\title{
Retraction Note: Effects of alendronate on metacarpal and lumbar bone mineral density, bone resorption, and chronic back pain in postmenopausal women with osteoporosis
}

\author{
Jun Iwamoto ${ }^{1} \cdot$ Tsuyoshi Takeda $^{1} \cdot$ Yoshihiro Sato $^{2} \cdot$ Mitsuyoshi Uzawa $^{3}$
}

Published online: 18 June 2021

(c) International League of Associations for Rheumatology (ILAR) 2021

\section{Retraction Note: Clin Rheumatol (2004) 23:383-389 https://doi.org/10.1007//10067-004-0881-z}

The Editor-in-Chief has retracted this article [1]. After publication, serious concerns were raised with respect to the ethical oversight, methodology and authorship. Keiyu Orthopaedic Hospital has not been able to confirm that appropriate ethics approval was obtained. The Editor-in-Chief no longer has confidence in this article. Jun Iwamoto has not responded to correspondence from the Editor-in-Chief or publisher about this retraction. The Editor-in-Chief and the publisher were not able to obtain current email addresses for Tsuyoshi Takeda and Mitsuyoshi Uzawa. Yoshiro Sato is deceased.

\section{Reference}

1. Iwamoto J, Takeda T, Sato Y, Uzawa M (2004) Effects of alendronate on metacarpal and lumbar bone mineral density, bone resorption, and chronic back pain in postmenopausal women with osteoporosis. Clin Rheumatol 23:383-389. https://link.springer. com/article/10.1007/s10067-004-0881-z

Publisher's note Springer Nature remains neutral with regard to jurisdictional claims in published maps and institutional affiliations.

The original article can be found online at https://doi.org/10.1007/ s10067-004-0881-z.

Jun Iwamoto

jiwamoto@sc.itc.keio.ac.jp

1 Department of Sports Medicine, Keio University, School of Medicine, 35 Shinanomachi, Shinjuku-ku, Tokyo 160-8582, Japan

2 Department of Neurology, Mitate Hospital, Fukuoka, Japan

3 Department of Orthopaedic Surgery, Keiyu Orthopaedic Hospital, Gunma, Japan 